PROCEEDINGS OF THE

AMERICAN MATHEMATICAL SOCIETY

Volume 131, Number 11, Pages 3411-3422

S 0002-9939(03)07043-6

Article electronically published on March 25, 2003

\title{
DISCRETE GROUPS ACTIONS AND CORRESPONDING MODULES
}

\author{
E. V. TROITSKY
}

(Communicated by David R. Larson)

\begin{abstract}
We address the problem of interrelations between the properties of an action of a discrete group $\Gamma$ on a compact Hausdorff space $X$ and the algebraic and analytical properties of the module of all continuous functions $C(X)$ over the algebra of invariant continuous functions $C_{\Gamma}(X)$. The present paper is a continuation of our joint paper with M. Frank and V. Manuilov. Here we prove some statements inverse to the ones obtained in that paper: we deduce properties of actions from properties of modules. In particular, it is proved that if for a uniformly continuous action the module $C(X)$ is finitely generated projective over $C_{\Gamma}(X)$, then the cardinality of orbits of the action is finite and fixed. Sufficient conditions for existence of natural conditional expectations $C(X) \rightarrow C_{\Gamma}(X)$ are obtained.
\end{abstract}

\section{INTRODUCTION}

Given a discrete countable group $\Gamma$ acting on a compact Hausdorff space $X$, one can talk about the following notions:

(1) The orbits (their cardinality and other dynamical properties).

(2) In some cases, using invariant means on functions on $\Gamma$, it is possible to define so-called conditional expectations $E_{\Gamma}: C(X) \rightarrow C_{\Gamma}(X)$ and study their properties.

(3) It is possible to consider properties of the module of all continuous functions $C(X)$ over the algebra of invariant continuous functions $C_{\Gamma}(X)$. In some cases with the help of $E_{\Gamma}$ it can be equipped with a $C_{\Gamma}(X)$-valued $\mathrm{C}^{*}$-inner product.

The study of the relations between these properties was started systematically in [5. (for preliminary and related research see also 1, 2, 4, 7, 8, 10, 14, 15, 18] and the overview in $[5]$ ).

In the present paper we prove some statements inverse to the ones obtained in the aforementioned paper: from some properties of modules (3) we deduce some properties of actions (1). In particular, it is proved that if for a uniformly continuous action the module $C(X)$ is finitely generated projective over $C_{\Gamma}(X)$, then the cardinality of orbits is finite and fixed.

Received by the editors October 8, 2001 and, in revised form, May 21, 2002.

2000 Mathematics Subject Classification. Primary 37Bxx, 46L08; Secondary 47B48.

Key words and phrases. Discrete groups, discrete noncommutative dynamical systems, Hilbert $\mathrm{C}^{*}$-modules.

This work was partially supported by the RFBR (Grant 02-01-00572) and by the President of RF (Grant 00-15-99263). 
Some sufficient conditions of the Lyapunov type for existence of natural conditional expectations $C(X) \rightarrow C_{\Gamma}(X)$ are obtained.

In the last section we give a description of the class of module-infinite algebras introduced in the previous sections.

\section{Preliminaries AND REMinding}

The necessary information about Hilbert $\mathrm{C}^{*}$-modules can be found in [11] and 12 .

Suppose $\Gamma$ is a discrete countable group and $X$ is a locally compact Hausdorff $\Gamma$ space. Let us denote by $M_{\Gamma}(f)$ or simply by $M(f)$ the invariant mean of a bounded function $f: \Gamma \rightarrow \mathbb{C}$ if it exists (if $\Gamma$ is amenable or $f$ is almost periodic [6]). Let $C_{\Gamma}(X)$ be the algebra of continuous invariant functions. The algebra of all continuous functions $C(X)$ is a module over $C_{\Gamma}(X)$. Let us denote by $L_{g}$ the left translation on functions on $\Gamma:\left(L_{g} \varphi\right)(h):=\varphi(g h)$.

Let us reformulate some definitions and statements from [5]. Actions will always be assumed to satisfy the following condition.

Definition 1 (5, Def. 2.1]). An action of a topological group $G$ on a locally compact Hausdorff space $X$ is uniformly continuous if for every point $x \in X$ and every neighborhood $U_{x}$ of $x$ there exists a neighborhood $V_{x}$ of $x$ such that $g\left(V_{x}\right) \subseteq$ $U_{x}$ for every $g \in G_{x}$, where $G_{x}$ is the isotropy subgroup at the point $x$.

Definition 2 (cf. [5 Def. 2.3]). Let us define a conditional expectation $E_{\Gamma}$ : $C(X) \rightarrow C_{\Gamma}(X)$ by the formula

$$
\left(E_{\Gamma}(\varphi)\right)(x):=M_{\Gamma}\left(\varphi_{x}\right), \quad \text { where } \quad \varphi_{x}(g):=\varphi(g x) .
$$

Remark 3. Of course, this expectation $E_{\Gamma}$ is not always defined. The obstructions are 1) existence of an averaging at each point, and 2) the resulting function $E_{\Gamma}(\varphi)$ can be discontinuous. In their absence one gets a linear mapping $E_{\Gamma}: C(X) \rightarrow$ $C_{\Gamma}(X)$. It is evident that since $M_{\Gamma}(1)=1$, this mapping is identical on invariant functions, hence a projection. The inequality ([6]) $\inf \{f(g)\} \leq M_{\Gamma}(f) \leq \sup \{f(g)\}$ shows that $\left\|E_{\Gamma}\right\|=1$ and so we really have a conditional expectation.

Remark 4. As was shown in [5 p. 840], in the case of finite orbits the averaging over orbits gives the same map $E_{\Gamma}$. Indeed, let $T:=\left\{g_{1}, \ldots, g_{N}\right\}$ be representatives of left cosets of $\Gamma_{x}$ in $\Gamma$. Then

$$
\begin{aligned}
M_{\Gamma}\left(\varphi_{x}\right) & =M_{\Gamma}\left(\sum_{g_{k} \in T} \varphi_{x}\left(g_{k}\right) \chi_{g_{k} H}\right)=\sum_{g_{k} \in T} \varphi\left(g_{k} x\right) M_{\Gamma}\left(\chi_{g_{k} H}\right) \\
& =\sum_{g_{k} \in T} \varphi\left(g_{k} x\right) M_{\Gamma}\left(\chi_{H}\right)=\mathrm{const} \cdot \sum_{g_{k} \in T} \varphi\left(g_{k} x\right),
\end{aligned}
$$

where $\chi$ are the characteristic functions. Taking $f=1$ we obtain const $=1 / N$. This is a proof for amenable groups. For an almost periodic function it is evident that the averaging over the finite orbit gives a constant function, which is a convex combination of $N$ translations of the original function.

Definition 5. If $E_{\Gamma}$ is well-defined one can equip $C(X)$ with the structure of a pre-Hilbert $\mathrm{C}^{*}$-module over $C_{\Gamma}(X)$ by the formula

$$
\langle\varphi, \psi\rangle_{E}:=E_{\Gamma}\left(\varphi^{*} \psi\right) .
$$


Remark 6. Let us recall that a conditional expectation $E: A \rightarrow B \subset A$ is said to be faithful if $E\left(x^{*} x\right)=0$ implies $x=0$ for $x \in A$. This is always true for $E_{\Gamma}$.

Proposition 7 ([5, Prop 1.1]). Let $A$ be a $C^{*}$-algebra and $E: A \rightarrow B \subseteq A$ be a conditional expectation with fixed point set $B$. There then exists a finite real number $K \geq 1$ such that the mapping $\left(K \cdot E-\mathrm{id}_{A}\right)$ is positive if and only if $E$ is faithful and the (right) pre-Hilbert $B$-module $\left\{A, E\left(\langle\cdot, \cdot\rangle_{A}\right)\right\}$ is complete with respect to the norm $\left\|E\left(\langle\cdot, \cdot\rangle_{A}\right)\right\|_{B}^{1 / 2}\left(\right.$ where $\langle a, b\rangle_{A}=a^{*} b$ for $\left.a, b \in A\right)$.

Theorem 8 (combination of [5, Lemmas 2.9 and 2.11 and Theorem 2.12]). Suppose a discrete group $\Gamma$ acts on a locally compact Hausdorff space $X$ in such a way that $k:=\max \{\#(\Gamma x): x \in X\}<+\infty$. Then $E_{\Gamma}$ is well-defined.

If $X$ is a normal space, then $K\left(E_{\Gamma}\right)=k$. Hence, by Proposition 7, $C(X)$ is a Hilbert $C^{*}$-module over $C_{\Gamma}(X)$.

Theorem 9 ([5, Theorem 3.6]). Suppose $X$ is a compact Hausdorff space, $\Gamma$ is a discrete group acting in a uniformly continuous manner on $X$, and all orbits consist of the same finite number of points. Then the Hilbert $C_{\Gamma}(X)$-module $\left\{C(X), E_{\Gamma}(\langle\cdot, \cdot\rangle)\right\}$ is finitely generated and projective.

A Hilbert $B$-module $M$ is called self-dual if there is a natural isomorphism of $M$ onto the module $M^{\prime}$ of all anti-B-linear bounded functionals on $M$. If $B$ is unital, then any finitely generated projective module is self-dual. If $M^{\prime \prime}=M$, then the module $M$ is called ( $B$-)reflexive.

Theorem 10 ([5, Theorem 3.9]). Let $X$ be a compact Hausdorff space and $\Gamma$ a discrete group acting on $X$ uniformly continuously. If there exists an integer $n$ such that the cardinalities of all orbits do not exceed $n$ and the number of orbits whose cardinality is strictly less than $n$ is finite, then the Hilbert $C_{\Gamma}(X)$-module $\left\{C(X), E_{\Gamma}(\langle.,\rangle).\right\}$ is $C_{\Gamma}(X)$-reflexive.

The following final form of the previous theorem was obtained by V. Seregin.

Theorem 11 ([17]). Let $X$ be a compact Hausdorff space and $\Gamma$ a discrete group acting in a uniformly continuous manner on $X$. If $\Gamma$ acts in such a way that the cardinalities of all orbits are uniformly bounded, then the Hilbert $C_{\Gamma}(X)$-module $\left\{C(X), E_{\Gamma}(\langle.,\rangle).\right\}$ is $C_{\Gamma}(X)$-reflexive.

From here on $X$ is compact and separable, hence metrizable and normal.

\section{INVERSE THEOREMS}

In this section we will obtain some theorems, inverse to the main statements of the previous section.

Let us recall the following statement.

Theorem 12. Let $Z$ be an infinite compact Hausdorff space. There is no (complete) Hilbert space structure on $C(Z)$ with the norm satisfying $\|f\|_{h} \leq\|f\|_{C(Z)}$.

Proof. By [3, Theorem II.2.5] the two norms have to be equivalent. But $C(Z)$ is reflexive if and only if $Z$ is finite [3, IV.13.15]. 
Theorem 13. Let $\Gamma$ be a discrete group acting uniformly continuously on a compact separable Hausdorff topological space $X$. Then the following properties are equivalent:

(i) cardinalities of all orbits are uniformly bounded;

(ii) $E_{\Gamma}$ is well-defined and there exists a finite real number $K \geq 1$ such that the mapping $\left(K \cdot E-\mathrm{id}_{A}\right)$ is positive;

(iii) $E_{\Gamma}$ is well-defined and the corresponding pre-Hilbert module is complete;

(iv) $E_{\Gamma}$ is well-defined and the corresponding pre-Hilbert module is reflexive.

Proof. (i) $\Rightarrow$ (iv) is Theorem 11.

(iv) $\Rightarrow$ (iii) is evident.

(ii) $\Leftrightarrow$ (iii) is Proposition 7 .

In order to prove (ii) $\Rightarrow(\mathrm{i})$, let us suppose the opposite.

Notice that if $Y \subset X$ is a closed invariant subspace, then the pre-Hilbert $C_{\Gamma}(Y)$ module $C(Y)$ is also complete (see Lemma 19).

Assume first that there is an infinite orbit $\Gamma x$. Taking $Y=\overline{\Gamma x}$ and applying the remark above we obtain that $C(Y)$ could be equipped with the Hilbert space structure, because $C_{\Gamma}(Y) \cong \mathbb{C}$. Contradiction with Theorem 12 .

The other case is the case of finite orbits of arbitrary cardinality. Here we can argue as in [5. Theorem 2.12]. By Proposition 7 there exists a real number $K \geq 1$ such that $K \cdot E_{\Gamma}(\varphi) \geq \varphi$ for any positive function $\varphi: X \rightarrow \mathbb{C}$. Choose a point $x \in X$ with $k_{x}:=\#(\Gamma x)>K$. Then we can obtain a neighborhood $U_{x}$ of $x$ such that $g_{i} U_{x} \cap g_{j} U_{x}=\emptyset$ for $i \neq j$ and $\left\{g_{1}=1, g_{2}, \ldots, g_{m}\right\}=T_{x}:=\Gamma / \Gamma_{x}$, where $\Gamma_{x}$ is the isotropy subgroup. For a continuous non-negative function $f$ with support inside $U_{x}$ (cf. [16, Th. V.17.4] for the existence) we have the inequality

$$
K \cdot E_{\Gamma}(f)(x)=K \frac{1}{k_{x}} \cdot \sum_{g_{a} \in T_{x}} f\left(g_{a} x\right)<f(x) .
$$

This contradicts the definition of $K$.

Theorem 14. Let $\Gamma$ be a discrete group acting uniformly continuously on a compact separable Hausdorff connected topological space X. Suppose that the averaging over orbits defines a structure of a self-dual Hilbert module on $C(X)$ over the algebra of invariant continuous functions $C_{\Gamma}(X)$. If all orbits are finite, then they have the same cardinality.

Proof. Let $W \subset X$ be the open subset consisting of all points from orbits of the maximal length $k$. Suppose $W \neq X$ and take $x \in \bar{W} \cap(X \backslash W)$ (since $X$ is connected, this is possible).

Then for any neighborhood $U$ of $x$ there exist $y \in U \cap W$ and $g \in \Gamma_{x}$ such that $g y \neq y$, where $\Gamma_{x}$ is the stabilizer of $x$. Indeed, suppose the opposite: there exists a neighborhood $U$ such that any element $y \in U \cap W$ is $\Gamma_{x}$ invariant, i.e. $\Gamma_{x} \subset \Gamma_{y}$. Then $\#(\Gamma x) \geq \#(\Gamma y)$, and we have a contradiction.

So, we can choose a sequence of elements $y_{n} \in W$ of different orbits (due to finiteness of orbits) and a sequence $g_{n} \in \Gamma_{x}$ such that $y_{n} \rightarrow x(n \rightarrow \infty)$ and $y_{n}^{\prime}:=g_{n} y_{n} \neq y_{n}$. Since $X$ is normal, we can choose (passing to a subsequence, if necessary) two sequences of open neighborhoods $W \supset V_{n} \ni y_{n}$ and $V_{n}^{\prime}=g_{n} V_{n} \ni y_{n}^{\prime}$ non-intersecting with each other and with $x$ and satisfying the following condition: for any neighborhood $V$ of $x$ the sets $V_{n}$ and $V_{n}^{\prime}$ are inside $V$ for all large $n$. Take 
continuous functions

$$
\begin{array}{cc}
\varphi_{n}: X \rightarrow[0,1], \quad \varphi_{n}\left(y_{n}\right) & =1, \quad \operatorname{supp} \varphi_{n} \subset V_{n}, \\
\varphi_{n}^{\prime}: X \rightarrow[-1,0], \quad \varphi_{n}^{\prime}\left(y_{n}^{\prime}\right)=-1, \quad \operatorname{supp} \varphi_{n}^{\prime} \subset V_{n}^{\prime}, \\
\varphi_{n}^{\prime}\left(g_{n} z\right)=-\varphi_{n}(z) .
\end{array}
$$

Define the function $\varphi: X \rightarrow[-1,1]$ to be equal to $\varphi_{n}$ on $V_{n}$, to $\varphi_{n}^{\prime}$ on $V_{n}^{\prime}$, and vanish in other points. It is continuous in all points except of $x$. Then $F(f):=$ $E_{\Gamma}(\varphi f)$ is a $C_{\Gamma}(X)$ functional on $C(X)$. It cannot be presented as $E_{\Gamma}(g f)$ with $g \in C(X)$. Indeed, the desired $g$ has to coincide with $\varphi$ on the open invariant set $W$. Contradiction with self-duality.

Lemma 15. Suppose $X$ is a compact normal space and $C(X)$ is a finitely generated module over $C_{\Gamma}(X)$. Then the cardinality of each orbit is finite.

Proof. Suppose there exists an infinite orbit. Then the dimension (algebraic) of the space of continuous functions on it (or its closure) is infinite. Hence it cannot be finitely generated over constants (invariant functions). By the Tietze theorem all of these functions are the restrictions of some continuous functions to the closure of the orbit. Hence the same statement is true for all functions.

Definition 16. A $\mathrm{C}^{*}$-algebra $A$ is called module-infinite (MI) if each countably generated Hilbert $A$-module is projective finitely generated if and only if it is selfdual. Let us remark that a projective finitely generated module over a unital algebra is always self-dual. We will give a description of MI algebras in Section 6 ]

Theorem 17. Let $\Gamma$ be a discrete group acting uniformly continuously on a compact separable Hausdorff connected topological space $X$. Suppose the averaging over orbits defines a structure of a self-dual Hilbert module on $C(X)$ over the algebra $C_{\Gamma}(X)$. If $C_{\Gamma}(X)$ is $\mathrm{MI}$, then all orbits have the same cardinality.

Proof. By Lemma 15 the orbits are finite. Suppose that there are orbits of cardinalities $k$ and smaller. Let $W \subset X$ be the open subset formed by all points from the orbits of cardinality $\geq k$. Then $W \neq X$ and we can take $x \in \bar{W} \cap(X \backslash W)$ (since $X$ is connected, it is possible). So $\#(\Gamma x)=m<k$. The proof could be completed as for Theorem 14 .

\section{LOCALIZATION AND GLOBALIZATION}

It turns out that we can deduce facts about cardinality of orbits from a purely algebraic data.

Let us start from the following evident lemma, which formulates an idea from the previous section in a more general way.

Lemma 18. Let $C(X)$ be m-generated over $C_{\Gamma}(X)$. If $Y$ is a closed $\Gamma$-invariant subspace of $X$, then $C(Y)$ is m-generated over $C_{\Gamma}(Y)$.

Lemma 19. Suppose $X$ is a compact separable Hausdorff space, $E_{\Gamma}$ is well-defined and the corresponding pre-Hilbert module is complete. If $Y \subset X$ is a closed invariant subspace, then the pre-Hilbert $C_{\Gamma}(Y)$-module $C(Y)$ is also complete.

Proof. By Proposition[7 there exists a real number $K \geq 1$ such that $K \cdot E_{\Gamma}(\varphi) \geq \varphi$ for any positive function $\varphi: X \rightarrow \mathbb{C}$. Let $\widehat{\varphi}: Y \rightarrow \mathbb{C}$ be a positive function. By the 
Tietze theorem, there is a positive continuous extension of $\widehat{\varphi}$ up to $\varphi: X \rightarrow \mathbb{C}$. We have

$$
K \cdot E_{\Gamma}(\varphi) \geq \varphi,\left.\quad K \cdot E_{\Gamma}(\varphi)\right|_{Y} \geq\left.\varphi\right|_{Y}, \quad K \cdot E_{\Gamma}(\widehat{\varphi}) \geq \widehat{\varphi} .
$$

Applying Proposition 7 in the opposite direction we complete the proof.

Lemma 20. If an orbit $Y=\Gamma x$ consists of exactly $k$ points, then $C(Y)$ is the free $k$-generated module over $C_{\Gamma}(Y)$.

Proof. $C_{\Gamma}(Y) \cong \mathbb{C}$ and $C(Y) \cong \mathbb{C}^{k}$ as a $\mathbb{C}$-module.

Lemma 21. If an orbit $Y=\Gamma x$ is infinite, then $C(\bar{Y})$ is not a finitely generated module over $C_{\Gamma}(\bar{Y})$.

Proof. $C_{\Gamma}(\bar{Y}) \cong \mathbb{C}$, and the algebra of continuous functions on an infinite compact Hausdorff space is $\mathbb{C}$-infinite.

We need the following statement.

Proposition 22. Let $C(X)$ be m-generated over $C_{\Gamma}(X)$, where $X$ is a compact separable Hausdorff space. Then the cardinality of each orbit does not exceed $m$.

Proof. The statement is a combination of Lemmas 18, 20, and 21.

Theorem 23. Suppose an action of discrete group $\Gamma$ on a compact separable Hausdorff space $X$ is uniformly continuous. Let $C(X)$ be a finitely generated projective module over $C_{\Gamma}(X)$. Then all orbits have the same finite cardinality.

Proof. By Proposition 22 the cardinality of orbits is bounded by $m$. Hence, by Theorem $8 E_{\Gamma}$ is well-defined and the pre-Hilbert module $C(X)$ over $C_{\Gamma}(X)$ is complete. Since it is finitely generated projective, it is a self-dual. So, we have both properties and we do not need MI to complete the proof as in Theorem 17

\section{EXISTENCE THEOREM}

From here on we will concentrate on some specific properties of an action of an (infinite) discrete group $G$ on a compact Hausdorff separable space $X$. Since such spaces are metrizable, we may fix a metric $\rho$ on $X$. Of course, the considerations below can be formulated using topology and neighborhoods instead of the distance and $\varepsilon$-neighborhoods.

In the present section we will obtain some sufficient conditions for $E_{\Gamma}$ to be well-defined.

Let us start with a few examples.

Example 24. Let $X \subset \mathbb{R}^{3}$ consist of 2 circles

$$
S_{ \pm}: \quad\left\{\begin{array}{l}
x=\cos 2 \pi t, \\
y=\sin 2 \pi t, \\
z= \pm 1,
\end{array} \quad t \in[0,1],\right.
$$

and non-uniform spiral

$$
\Sigma: \quad\left\{\begin{array}{l}
x=\cos 2 \pi \tau, \\
y=\sin 2 \pi \tau, \\
z=\frac{2}{\pi} \cdot \arctan \tau,
\end{array} \quad \tau \in[0,1] .\right.
$$


Let the generator $g$ of $\Gamma=\mathbb{Z}$ act on all three components by

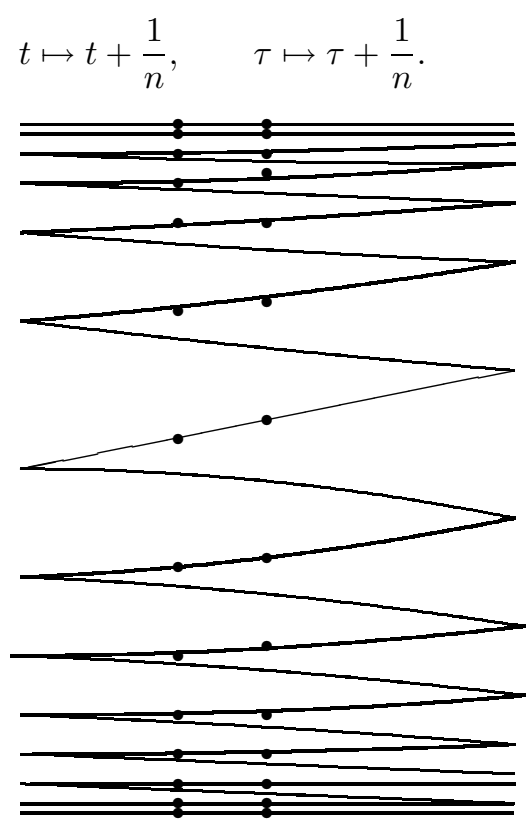

Since $\mathbb{Z}$ is amenable, the pointwise value of $E_{\Gamma}$ is defined. If $\varphi: X \rightarrow \mathbb{R}_{+} \subset \mathbb{C}$ is a non-negative continuous function with

$$
\left.\varphi\right|_{S_{+}}=0,\left.\quad \varphi\right|_{\Sigma}=1 \quad \text { for } \quad t \leq 0,\left.\quad \varphi\right|_{S_{-}}=1,
$$

then $\left.E_{\Gamma}(\varphi)\right|_{S_{+}}=0$ and $\left.E_{\Gamma}(\varphi)\right|_{\Sigma}>0$, so that the function $E_{\Gamma}(\varphi)$ is discontinuous.

In this example the action is not uniformly continuous and we always emphasized that these are the bad ones. Unfortunately, the next example shows that the condition on action to be uniformly continuous does not help much either.

Example 25. Let $X$ and $\Gamma$ be as in the previous example, but the action be defined by

$$
t \mapsto t+\alpha, \quad \tau \mapsto \tau+\alpha,
$$

where $\alpha$ is a positive irrational number. Then the isotropy group of each point of $X$ is trivial. Hence, the condition of uniform continuity holds automatically. Nevertheless, a discontinuous $E_{\Gamma}(\varphi)$ corresponds to the same function $\varphi$.

Remark 26. In any of these examples let $x \in \Sigma$. Then for the aforementioned $\varphi$ the function $\varphi_{x}: \mathbb{Z} \rightarrow \mathbb{C}$ is not almost periodic. Of course, for amenable $\Gamma=\mathbb{Z}$ this is not an obstruction, but a similar phenomenon can occur for non-amenable groups, too.

Now we introduce a condition which is sufficient to overcome these difficulties.

Definition 27. An action of a group $\Gamma$ on a metric space $X$ is called (Lyapunov) stable if for any $\varepsilon>0$ and any $x \in X$ there exist $\delta>0$ such that

$$
\rho(g x, g y)<\varepsilon \text { for any } g \in \Gamma \text { if } \rho(x, y)<\delta .
$$


The following statement is evident.

Proposition 28. If an action is stable, then it is uniformly continuous.

Proposition 29. Let a discrete group $\Gamma$ act on a compact separable Hausdorff space $X$ in a stable way. If $\varphi: X \rightarrow \mathbb{C}$ is a continuous function, then for any $x \in X$ the function $\varphi_{x}: \Gamma \rightarrow \mathbb{C}, \varphi_{x}(g):=\varphi(g x)$, is almost periodic, and therefore its mean $M\left(\varphi_{x}\right)$ is well-defined.

Proof. Let $\varepsilon>0$ be arbitrary. Since $X$ is compact and $\varphi$ is continuous, it is uniformly continuous, in particular, there exists $\widetilde{\varepsilon}$ such that

$$
|\varphi(y)-\varphi(z)|<\varepsilon \quad \text { if } \rho(z, y)<\widetilde{\varepsilon} .
$$

For each point $g x$ of the orbit let us find an $\varepsilon_{g}$-neighborhood $U_{g}$ with the following property:

$$
\rho(h g x, h y)<\widetilde{\varepsilon} \quad \text { for any } h \in \Gamma \text { if } y \in U_{g} .
$$

Let $U_{g_{1}}, \ldots, U_{g_{m}}$ be a finite subcovering of the (closure of) the orbit $\Gamma x$. Then the functions $L_{g_{s}} \varphi_{x}, s=1, \ldots, m$, form an $\varepsilon$-net for the set $L_{g} \varphi_{x}, g \in \Gamma$, with respect to the uniform norm. Indeed, for any $g \in \Gamma$ we can choose $s_{0} \in\{1, \ldots, m\}$ such that $y=g x \in U_{g_{s_{0}}}$. Then by (11) and (2) we have

$$
\sup _{h \in \Gamma}\left|\left(L_{g} \varphi_{x}\right)(h)-\left(L_{g_{s_{0}}} \varphi_{x}\right)(h)\right|=\sup _{h \in \Gamma}\left|\varphi(h g x)-\varphi\left(h g_{s_{0}} x\right)\right|<\varepsilon .
$$

Theorem 30. Let a discrete group $\Gamma$ act on a compact separable Hausdorff space $X$. If the action is stable, then $E_{\Gamma}$ is well-defined.

Proof. By Proposition 29 we only need to verify continuity of the mean $M\left(\varphi_{x}\right)$ in $x \in X$. Let $x \in X$ and $\varepsilon>0$ be arbitrary. Let us recall (cf. [6. pp. 250-251]) that we can choose $h_{1}, \ldots, h_{p} \in \Gamma$ in such a way that the uniform distance on $\Gamma \times \Gamma$ between the function

$$
\frac{1}{p} \sum_{j=1}^{p} D_{h_{j}} \varphi_{x}: \Gamma \times \Gamma \rightarrow \mathbb{C} \quad\left(\text { where } \quad\left(D_{h} \psi\right)\left(g_{1}, g_{2}\right):=\psi\left(g_{1} h g_{2}\right)\right)
$$

and some constant is less than $\varepsilon$. In this case the uniform distance satisfies the inequality

$$
\left\|M\left(\varphi_{x}\right)-\frac{1}{p} \sum_{j=1}^{p} D_{h_{j}} \varphi_{x}\right\|_{u}<2 \varepsilon
$$

Let us choose a $\delta$-neighborhood $U$ of $x$ such that

$$
|\varphi(g y)-\varphi(g x)|<\varepsilon, \quad \text { for any } g \in \Gamma, y \in U \text {. }
$$

This neighborhood can be found as in the proof of Proposition 29; $\varepsilon \rightarrow \widetilde{\varepsilon} \rightarrow \delta$. More precisely, first we can find $\widetilde{\varepsilon}>0$ such that $|\varphi(y)-\varphi(z)|<\varepsilon$ whenever $\rho(y, z)<\widetilde{\varepsilon}$ (using compactness of $X$ ). Then, by stability of the action, we can find for our fixed $x$ a number $\delta>0$ such that $\rho(g y, g x)<\widetilde{\varepsilon}$ for any $g \in \Gamma$ whenever $\rho(y, x)<\delta$. 
Then for any $y \in U$ one has

$$
\begin{aligned}
\left(\frac{1}{p} \sum_{j=1}^{p} D_{h_{j}} \varphi_{y}\right)\left(g_{1}, g_{2}\right) & \\
& =\left(\frac{1}{p} \sum_{j=1}^{p} D_{h_{j}} \varphi_{x}\right)\left(g_{1}, g_{2}\right)+\frac{1}{p} \sum_{j=1}^{p}\left(\varphi_{y}\left(g_{1} h_{j} g_{2}\right)-\varphi_{x}\left(g_{1} h_{j} g_{2}\right)\right) \\
& =\left(\frac{1}{p} \sum_{j=1}^{p} D_{h_{j}} \varphi_{x}\right)\left(g_{1}, g_{2}\right)+\frac{1}{p} \sum_{j=1}^{p}\left(\varphi\left(g_{1} h_{j} g_{2} y\right)-\varphi\left(g_{1} h_{j} g_{2} x\right)\right) .
\end{aligned}
$$

Each term of the second summand is less than $\varepsilon$. Hence, the second summand is less than $\varepsilon$. Thus,

$$
\left\|M\left(\varphi_{x}\right)-\frac{1}{p} \sum_{j=1}^{p} D_{h_{j}} \varphi_{x}\right\|_{u}<3 \varepsilon .
$$

Therefore, considering $M\left(\varphi_{x}\right)$ as an arbitrary constant, we have

$$
\left\|M\left(\varphi_{y}\right)-\frac{1}{p} \sum_{j=1}^{p} D_{h_{j}} \varphi_{x}\right\|_{u}<6 \varepsilon .
$$

Finally,

$$
\left|M\left(\varphi_{y}\right)-M\left(\varphi_{x}\right)\right|<9 \varepsilon
$$

\section{Commutative Mi algebras}

Definition 31. A commutative unital $C^{*}$ algebra $A=C(Y)$ is said to be DI (divisible infinite) if for any infinite sequence $u_{i}$ of its elements of norm $1 \geq\left\|u_{i}\right\| \geq$ $C>0$ there exist a subsequence $i(k)$ and elements $0 \leq b_{k} \in A$ of norm 1 , such that

(i) the supports of $b_{k}$ in $Y$ are pairwise disjoint, and

(ii) for each $k$ there exist points $y_{k}, y_{k}^{\prime}$ such that $b_{k}\left(y_{k}\right)=1, y_{k}^{\prime} \notin \bigcup_{j} \operatorname{supp} b_{j}$, $\left|u_{i(k)}\left(y_{k}^{\prime}\right)\right| \geq \delta,\left|u_{i(k)}\left(y_{k}\right)\right| \geq \delta$, and the sequences $\left\{y_{k}\right\}$ and $\left\{y_{k}^{\prime}\right\}$ have a common accumulation point. In particular, $\sum_{k} b_{k}^{s}$ is a discontinuous function for any integer $s \geq 1$.

Theorem 32. If a commutative unital $C^{*}$-algebra $A$ is DI, then it is MI.

Proof. We have to prove that any countable generated self-dual Hilbert $A$-module $\mathcal{M}$ is finitely generated projective. By the Kasparov stabilization theorem [9] (see also [11, 12]) one has $\mathcal{M} \oplus l_{2}(A) \cong l_{2}(A)$, where $l_{2}(A)$ is the standard Hilbert module (see, e.g. [12]). Denote by $p: l_{2}(A) \rightarrow \mathcal{M} \subset l_{2}(A)$ the corresponding orthoprojection. Let $p_{j}: l_{2}(A) \rightarrow E_{j} \cong A^{j}$ be the orthoprojection on the first $j$ standard summands of $l_{2}(A)$. Two opportunities can arise: 1$)\left\|\left(1-p_{j}\right) p\right\| \rightarrow 0$ as $j \rightarrow \infty$, and 2) the opposite case.

1) Let us show that in this case $\mathcal{M}$ is finitely generated projective. One can argue as in [13]: for a sufficiently large $j$ the operator

$$
J(x)= \begin{cases}p_{j}(x) & \text { if } x \in \mathcal{M} \\ x & \text { if } x \in \mathcal{M}^{\perp} \cong l_{2}(A),\end{cases}
$$


is close to identity, hence an isomorphism. It maps $\mathcal{M}$ isomorphically onto a direct summand of $E_{j}$.

2) In this case (changing the standard decomposition, if necessary) we can find a sequence $j(k)$ such that for each $k$ there exists an element $z_{k} \in \mathcal{M}$ of norm 1 such that its $j(k)$-th entry $z_{k}^{j(k)}$ with respect to the standard decomposition has the norm greater than some fixed $C>0$. According to Definition 31 let us choose functions $b_{k}$ (for the sake of notational brevity we assume that we do not need to pass to a subsequence the second time). Then the formula $\beta(x)=\sum_{k} b_{k} x^{j_{k}}$ defines a functional on $l_{2}(A)$. It is evident that it does not admit an adjoint on $l_{2}(A)$, because $b_{k} \nrightarrow 0$ as $k \rightarrow \infty$. Let us show that there is no adjointable functional $\alpha$ on $l_{2}(A)$ such that $\left.\alpha\right|_{\mathcal{M}}=\left.\beta\right|_{\mathcal{M}}$, and hence $\left.\beta\right|_{\mathcal{M}}$ is a non-adjointable functional on $\mathcal{M}$ and $\mathcal{M}$ is not a self-dual module. Indeed, suppose there exists an element $a=\left(a_{1}, a_{2}, \ldots\right) \in \mathcal{M} \subset l_{2}(A)$ such that $\alpha(x):=\sum_{i} a_{i} x^{i}=\beta(x)$ for any $x \in \mathcal{M}$. Then

$$
\langle a, a\rangle \leq \sum_{k} b_{k}^{2}
$$

(the last element is a bounded measurable, but discontinuous function; see Definition 31(ii)), because for any continuous positive function $f_{k}$, which is equal to 1 on $\bigcup_{j \leq k} \operatorname{supp} b_{j}$ and 0 on $\bigcup_{j>k} \operatorname{supp} b_{j}$ (the existence follows from normality of $Y$ ), one has $\beta f_{k}=\alpha f_{k}+(\beta-\alpha) f_{k}$. But $\beta f_{k}$ is an element of $l_{2}(A)$ (or, more precisely, an adjointable functional $\left.\beta f_{k}(x)=\sum_{j \leq k} b_{k} x^{j_{k}}\right)$. Let $b f_{k}$ be the corresponding element. Then $b f_{k}=a f_{k}+(b-a) f_{k}$ is the decomposition corresponding to $l_{2}(A)=\mathcal{M} \oplus l_{2}(A)$. Indeed, $a \in \mathcal{M}$, and for any $x \in M,\left\langle(b-a) f_{k}, x\right\rangle=0$, because $\alpha(x)=\beta(x)$ for those $x$. The Pythagorean theorem for Hilbert modules shows that $\langle a, a\rangle f_{k}^{2} \leq \sum_{j \leq k} b_{j}^{2}$. Taking all $f_{k}$ 's one obtains (3).

On the other hand,

$$
\langle a, a\rangle\left(y_{i}\right) \geq\left\langle a, z_{i}\right\rangle\left\langle z_{i}, a\right\rangle\left(y_{i}\right)=\sum_{m, k} b_{m} z_{i}^{j(m)} \overline{z_{i}^{j(k)}} b_{k}\left(y_{i}\right)=b_{i}^{2}\left(y_{i}\right) z_{i}^{j(i)}\left(y_{i}\right) \geq \delta^{2} .
$$

But from (3) one obtains $\langle a, a\rangle\left(y_{i}^{\prime}\right)=0$. Hence, $\langle a, a\rangle$ does not belong to $A$. A contradiction.

Now we describe consequences of this fact for the case studied in the present paper.

Theorem 33. A commutative separable unital $C^{*}$-algebra $A$ is MI if and only if its Gelfand spectrum $Y$ has no isolated points.

Proof. If $Y$ has an isolated point, a separable Hilbert space arises as one of self-dual modules, hence, MI does not hold.

Now, suppose $Y$ has no isolated points; in particular it is infinite. Since $Y$ is a compact Hausdorff separable space, the topology is generated by some metric $\rho$. For any given sequence $u_{i}$ of norm $\geq C$ we can find a sequence of different points $\widetilde{y}_{i}$ such that $\left|u_{i}\left(\widetilde{y}_{i}\right)\right|>2 C / 3$.

Since $Y$ is compact, one can pass to a convergent subsequence $y_{k}=\widetilde{y}_{i(k)}$. For a convergent sequence by induction we can choose $\varepsilon_{k}$ such that the corresponding $\varepsilon_{k}$-neighborhoods $U_{k}$ of $y_{k}$ are pairwise disjoint. Then we can choose $y_{k}^{\prime} \neq y_{k}$ 
inside these neighborhoods so close to $y_{k}$ that $\left|u_{i(k)}\right| \geq C / 2$. After that one can choose functions $b_{k}$ such that $b_{k}\left(y_{k}\right)=1$ and $\operatorname{supp} b_{k} \subset U_{k} \backslash y_{k}^{\prime}$. It remains to take $\delta=C / 2$.

Corollary 34. Suppose $X$ is a compact connected separable Hausdorff $\Gamma$-space. Then $C_{\Gamma}(X)$ is $\mathrm{MI}$ if and only if $X / \Gamma$ has at least two separated points.

Proof. The Gelfand spectrum $Y$ of $C_{\Gamma}(X)$ is a quotient space of $X / \Gamma$ with respect to the equivalence of non-separated points. Since $X$ is connected, $Y$ is connected, too. So, by Theorem [33, $C_{\Gamma}(X)$ is $\mathrm{MI}$ if and only if $Y$ is not reduced to one point.

\section{ACKNOWLEDGMENT}

Most of the present results were obtained due to the creative and helpful atmosphere of the Max-Planck-Institut für Mathematik (Bonn) during my visits in Fall 2000 and in Spring 2001.

I am grateful to M. Frank for initiating my work in this area and for sharing his knowledge of this field. I am also grateful to V. Manuilov for helpful discussions and suggestions at different stages of the present research.

I ams also grateful to the referee for very valuable remarks and suggestions.

\section{REFERENCES}

1. E. Andruchow and D. Stojanoff, Geometry of conditional expectations of finite index, Internat. J. Math. 5 (1994), 169-178. MR 95e:46085

2. M. Baillet, Y. Denizeau, and J.-F. Havet, Indice d'une esperance conditionelle, Compos. Math. 66 (1988), 199-236. MR 90e:46050

3. N. Dunford and J. T. Schwartz, Linear operators. I. General theory, Interscience, New York, 1958. MR 22:8302

4. M. Frank and E. Kirchberg, On conditional expectations of finite index, J. Oper. Theory 40 (1998), no. 1, 87-111. MR 2000k:46080

5. M. Frank, V. M. Manuilov, and E. V. Troitsky, On conditional expectations arising from group actions, Zeitschr. Anal. Anwendungen 16 (1997), 831-850. MR 2000e:46089]

6. E. Hewitt and K. A. Ross, Abstract harmonic analysis. I, Springer-Verlag, New York, 1963. MR 28:158

7. P. Jolissaint, Indice d'esperances conditionnelles et algèbres de von Neumann finies, Math. Scand. 68 (1991), 221-246. MR 93g:46059

8. V. Jones, Index of subfactors, Invent. Math. 41 (1981), 1-25. MR 84d:46097

9. G. G. Kasparov, Hilbert $C^{*}$-modules: theorems of Stinespring and Voiculescu, J. Operator Theory 4 (1980), 133-150. MR 82b:46074

10. M. Khoshkam, Hilbert $C^{*}$-modules and conditional expectations on crossed products, J. Austral. Math. Soc. (Series A) 61 (1996), 106-118. MR 97i:46100

11. E. C. Lance, Hilbert $C^{*}$-modules - a toolkit for operator algebraists, London Mathematical Society Lecture Note Series, vol. 210, Cambridge University Press, England, 1995. MR 96k: 46100

12. V. M. Manuilov and E. V. Troitsky, Hilbert $C^{*}$ - and $W^{*}$-modules and their morphisms, J. Math. Sci. (New York) 98 (2000), no. 2, 137-201. MR 2001k:46094

13. A. S. Mishchenko and A. T. Fomenko, The index of elliptic operators over $C^{*}$-algebras, Izv. Akad. Nauk SSSR, Ser. Mat. 43 (1979), 831-859, English translation, Math. USSR-Izv. 15, 87-112, 1980. MR 81i:46075

14. M. Pimsner and S. Popa, Entropy and index for subfactors, Ann. Scient. Ec. Norm. Sup. 19 (1986), 57-106. MR 87m:46120

15. M. Rieffel, Integrable and proper action on $C^{*}$-algebras, and square-integrable representations of groups, E-print, 1998.

16. W. Rinow, Lehrbuch der Topologie, Dt. Verlag Wiss., Berlin, 1975. MR 58:24157 
17. V. Seregin, Reflexivity of $C^{*}$-Hilbert modules arising from group actions, Moscow Univ. Math. Bull. (2002), to appear.

18. Y. Watatani, Index for $C^{*}$-subalgebras, Memoirs Amer. Math. Soc., vol. 424, AMS, Providence, 1990. MR 90i:46104

Department of Mechanics and Mathematics, Moscow State University, 119899 Moscow, Russia

E-mail address: troitsky@mech.math.msu.su

$U R L:$ http://mech.math.msu.su/ troitsky 